\title{
A GAS KINETIC SCHEME FOR HYBRID SIMULATION OF PARTIALLY RAREFIED FLOWS
}

\section{S. Colonia, R. Steijl, and G. Barakos}

\author{
School of Engineering, University of Liverpool
} Liverpool L69 3GH, U.K.

\begin{abstract}
Approaches to predict flow fields that display rarefaction effects incur a cost in computational time and memory considerably higher than methods commonly employed for continuum flows. For this reason, to simulate flow fields where continuum and rarefied regimes coexist, hybrid techniques have been introduced. In the present work, analytically defined gas-kinetic schemes based on the Shakhov and Rykov models for monoatomic and diatomic gas flows, respectively, are proposed and evaluated with the aim to be used in the context of hybrid simulations. This should reduce the region where more expensive methods are needed by extending the validity of the continuum formulation. Moreover, since for high-speed rarefied gas flows it is necessary to take into account the nonequilibrium among the internal degrees of freedom, the extension of the approach to employ diatomic gas models including rotational relaxation process is a mandatory first step towards realistic simulations. Compared to previous works of $\mathrm{Xu}$ and coworkers, the presented scheme is defined directly on the basis of kinetic models which involve a Prandtl number correction. Moreover, the methods are defined fully analytically instead of making use of Taylor expansion for the evaluation of the required derivatives. The scheme has been tested for various test cases and Mach numbers proving to produce reliable predictions in agreement with other approaches for near-continuum flows. Finally, the performance of the scheme, in terms of memory and computational time, compared to discrete velocity methods makes it a compelling alternative in place of more complex methods for hybrid simulations of weakly rarefied flows.
\end{abstract}

\section{NOMENCLATURE}

$c_{x} \quad$ Particle velocity in $x$-direction

$c_{x}^{\prime} \quad c_{x}-u_{x}$, particle velocity fluctuation

$f$ particle distribution function

F $\quad m f$

$m$ molecular mass

(C) The authors, published by EDP Sciences. This is an Open Access article distributed under the terms of the Creative Commons Attribution License 4.0 (http://creativecommons.org/licenses/by/4.0/). 
$p \quad \rho T$ (nondimensional equilibrium pressure)

$p_{r} \quad \rho T_{r}$ (nondimensional rotational pressure)

$p_{t} \quad \rho T_{t}$ (nondimensional translational pressure)

$q_{x} \quad$ Total heat flux in $x$-direction

$q_{x}^{r} \quad$ Rotational heat flux in $x$-direction

$q_{x}^{t} \quad$ translational heat flux in $x$-direction

$R$ gas constant

$t \quad$ Time variable

$T \quad$ Total temperature

$T_{r} \quad$ Rotational temperature

$T_{t} \quad$ translational temperature

$u_{x} \quad$ velocity in $x$-direction

W conservative variables vector

$\mathbf{x}$ spatial coordinates vector

$Z_{r} \quad$ collision number

$\Delta c_{x} \quad$ velocity step

$\Delta t \quad$ time step

$\Delta x \quad$ Spatial step

$\zeta \quad$ Rotational degrees of freedom

$\boldsymbol{\Psi}_{0} \quad\left(1, c_{x}, \mathbf{c}^{2}, 0\right)^{\mathrm{T}}$

$\Psi_{1} \quad(0,0,1,1)^{\mathrm{T}}$

$\lambda \quad$ Particle mean free path

$\mu \quad$ Viscosity

$\rho \quad$ Density

$\tau \quad$ particle collision time

$\phi \quad$ generic macroscopic variable

$\hat{\phi} \quad$ nondimensional generic macroscopic variable

\section{ACRONYMS}

AUSM Advection Upstream Splitting Method

BGK Bhatnagar-Gross-Krook

BTE Boltzmann Transport Equation

CE Chapman-Enskog

DSMC Direct Simulation Monte Carlo

DVM Discrete Velocity Method

ES Ellipsoidal-Statistical

GKS Gas-Kinetic Scheme

GKS $\tau^{*} \quad$ GKS with modified $\tau$

MD Molecular Dynamics

MPC Modular Particle-Continuum

MPI Message Passing Interface 


$\begin{array}{ll}\text { M } \Phi C & \text { Multiphysics Code } \\ \text { NS } & \text { Navier-Stokes } \\ \text { TVD } & \text { Total Variation Diminishing } \\ \text { UGKS } & \text { Unified GKS }\end{array}$

\section{INTRODUCTION}

At intermediate altitudes $(70-90 \mathrm{~km})$, the flow around hypersonic aircraft can be characterized as mainly continuum with localized areas (generated by the rapid expansion in the wake of the vehicle as well as by strong gradients in shock waves and boundary layers) that display rarefaction effects. The flow conditions near the vehicle surface and in the wake determine the drag and the heat transferred to the vehicle and its payload. Therefore, it is important that these regions are simulated using appropriate physical models. When the gradients of the macroscopic variables become so steep that their length scale is of the same order as the average distance travelled by molecules between collisions, the number of impacts is not enough to drive the fluid towards a local thermodynamic equilibrium. At these conditions, the flow can no longer be considered a continuum and the transport terms in the Navier-Stokes (NS) equations fail since the constitutive relation is not valid.

The mathematical model at molecular level is the Boltzmann transport equation (BTE) [1] and for the regions of the flow field where highly nonequilibrium effects occur, the direct simulation Monte Carlo (DSMC) method [2] is typically employed to statistically estimate the solution of the BTE. Alternatively, a discrete velocity method (DVM) $[3,4]$ can be used to solve a kinetic model approximation of the BTE [5-7].

In previous works [8-11], hybrid techniques have been introduced to simulate flow fields where continuum and rarefied regimes coexist. In these methods, the more expensive approaches, such as DSMC or DVM, are employed only where needed and is coupled with a finite-volume scheme for the NS equations used where the flow is continuum. A hybrid technique couples two simulation methods by means of information exchange between the parts of the flow domain. In recent works, this has been achieved using an overlap region where flow state variables or numerical fluxes are exchanged between the two models [12] or employing a buffer region where the two models are blended at equation level [13]. Recent works on these methods focused on rarefied high-Mach flow can be found in $[14-16]$.

An alternative approach is the unified gas-kinetic scheme (UGKS) [17, 18] which uses a finite-volume method where the numerical fluxes are based on the solution of the Shakhov model [6] for a monoatomic gas or the Rykov model [7] for a diatomic gas with rotational nonequilibrium. Where the flow is underresolved, by accounting for the pressure jump in the definition of the collision 
time, additional numerical viscosity is added resulting in a shock thickness of the order of the cell size [19]. This allows the UGKS to simulate flows in both rarefied and continuum regimes.

In the present work, two GKS methods, analytically-defined on the basis of the Chapman-Enskog (CE) expansion of nondimensional Shakhov and Rykov models, are proposed for the simulation of weakly rarefied flows. The derivatives of the equilibrium function and the time derivatives of the primitive variables are analytically defined, employing the compatibility condition of the kinetic model for the latter. In previous works from Xu and coworkers [20,21], similar GKS are defined using the CE solution of the Bhatnagan-Gross-Krook (BGK) model with rotational nonequilibrium and a scaling of the energy numerical flux [19] to correct the Prandtl number. Moreover, in those schemes, the required derivatives are expressed in terms of Taylor series where the coefficient are calculated by means of properties of the employed BGK model. The proposed GKS, due to the use of the CE expansion, is limited to near-continuum regions but is simpler than the UGKS $[17,18]$. However, the validity of the approach can be extended considering a modified collision time [22]. Also, this correction, in the present work, is defined fully analytically for both schemes.

Based on a literature survey of related works, the authors believe that the proposed GKS represents an efficient method, relative to DVM, capable of modeling complex diatomic gas flows with moderate rarefaction effects but with significant rotational nonequilibrium. As such, the proposed approach is a novel alternative to the DVM for a range of practically relevant flows. Moreover, the update of the nonequilibrium distribution function, as used in the UGKS, is neglected reducing the memory cost of the approach. Thus, the GKS method represents a viable option to reduce the cost of hybrid simulations by reducing the region where the expensive method is needed and extending the validity of the continuum formulation.

The schemes are built in a computational framework described in section 2 that also includes a DVM for the kinetic Boltzmann equations successfully employed for different monoatomic cases [23]. The framework has been recently improved [24] with the addition of the Rykov model and an Ellipsoidal-Statistical (ES) model [25] for diatomic gases with rotational nonequilibrium. In section 3, the mathematical definition of the Rykov model and of the proposed GKS are described. Finally, in section 4, some preliminary results where the presented GKS is coupled with a DVM are shown and an assessment of reliability and performance of the GKS is presented.

\section{MULTIPHYSICS CODE}

The methods used in the present work are built in the multiphysics code $(M \Phi C)$ developed at the University of Liverpool $[23,26,27]$. Multiphysics code is a com- 
putational framework designed for simulations of complex flows, where different mathematical flow models are employed for different regions of the flow domain depending on the flow physics. The NS equations represent the baseline level of mathematical models used. For the continuum flow solver based on the compressible NS equations, a cell-centered block-structured finite-volume method is employed using the $\mathrm{AUSM}^{+} / \mathrm{up}$ (advection upstream splitting method) for the convective fluxes [28]. For low-speed flow analysis, the framework further includes a Lattice Boltzmann Method as well as a Vortex-In-Cell method for vortex-dominated incompressible flows.

In the present work, emphasis is placed on the simulation of hypersonic, partially-rarefied flows for which mathematical models at a more detailed level of physics than the compressible NS equations are required. For flows with strong nonequilibrium and rarefied effects, the framework includes Molecular Dynamics (MD) methods as well as deterministic DVM for a range of kinetic Boltzmann equations. The Shakhov and ES models are included for monoatomic gas flows, while the Rykov model and a polyatomic ES-BGK model were implemented for diatomic gas flow simulations [24]. The kinetic models are discretized using a discrete velocity method within a finite-volume method for multiblock structured grids; second order total variation diminishing (TVD) time marching is employed. The velocity (phase) space is discretized using either a uniformly spaced method with the trapezoidal rule for the evaluation of the moments of the distribution functions or a Gauss-quadrature method with modified Hermite polynomials. For the present high-speed flow cases, the uniform velocity space with the trapezoidal rule is the preferred approach and was used exclusively.

The memory and CPU time requirements are considerable and for this reason, an efficient parallel implementation involving 'two' levels of parallelism was conceived. In this parallelization, the phase space as well as the flow domain are distributed over the processes. First, the phase space is partitioned in regular subspaces, each to be assigned to separate processes within separate message passing interface (MPI) communicators. The overall number of processes is then divided by the number of partitions to obtain the required number of communicators. The mesh-blocks in physical space are then distributed over these communicators to obtain an equal distribution of the cells. An important factor in the performance is the number of processes assigned to the velocity space discretization. Limiting the size of these communicators will minimize the overhead in collective operations required for the evaluations of the moments of the distribution functions. However, this implies an increasing number of communicators over which the mesh-blocks are divided, potentially creating a load imbalance in physical space. The best performance is obtained if the number of velocity space partitions is chosen such that the load imbalance in physical space is typically less than $10 \%$.

The parallel performance of the coupled continuum/kinetic solver was investigated as part of a European Union funded project (PRACE Preparatory 


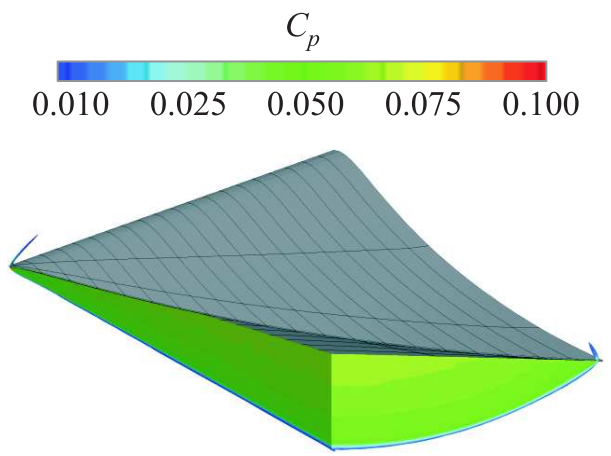

(a)

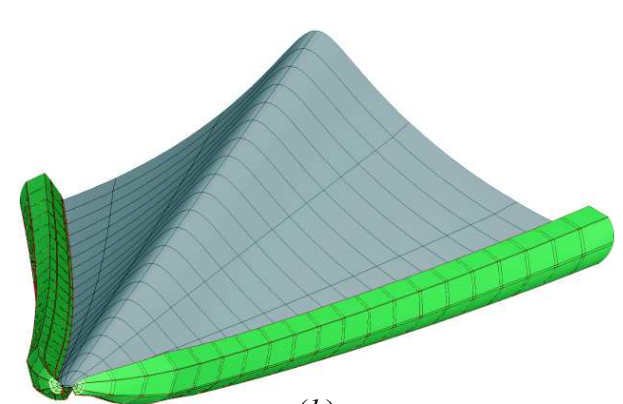

(b)

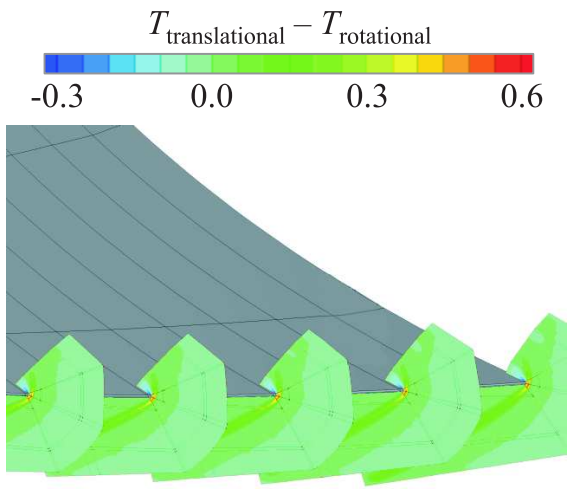

(c)

Figure 1 Pressure distribution at Mach $8(a)$; hybrid setup, kinetic-flow domains around leading-edges $(b)$; and flow solution in kinetic-flow domain $(c)$ at Mach 8 waverider, length $=5 \cdot 10^{3} \lambda$, Radius LE $=2.5 \lambda, T_{\infty}=116 \mathrm{~K}, T_{\text {wall }}=2.5 T_{\infty}, Z_{r}$ is determined by Eq. (4), $\mu$ is determined by Eq. (6), and specular wall boundary condition 


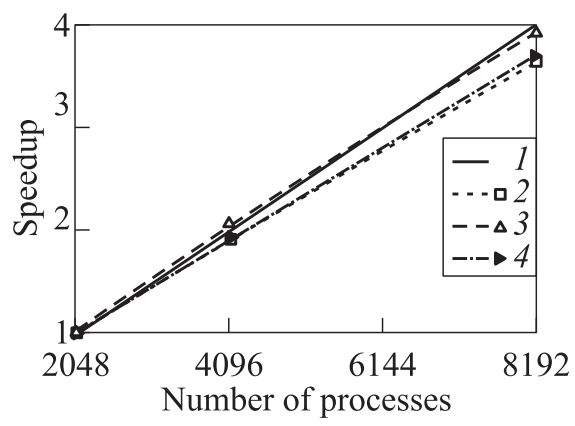

Figure 2 Strong scaling on SuperMUC (LRZ, Munich) at the conditions of Fig. 1: 1 - linear; 2 and 3 - full leading-edge, thin layer, velocity space discretization $32^{3}$ divided in $8 \times 4 \times 4$ and $8 \times 8 \times 4$ partitions, respectively; and 4 - full leading-edge, thick layer, velocity space discretization $24^{3}$ divided in $8 \times 4 \times 4$ partitions

Access), providing access to the SuperMUC computer at the Leibniz Rechenzentrum (LRZ) in Munich. In the project, 2048 to 8192 cores of Intel ${ }^{\circledR}$ Xeon ${ }^{\circledR}$ processors were used for a range of test cases. The example shown here is for the Mach 8 flow of a diatomic gas around the waverider geometry (leading-edge diameter $5 \lambda$ and body length $5 \cdot 10^{3} \lambda$ ) shown in Fig. 1a. A multiblock mesh with 867 blocks and $3 \cdot 10^{6}$ cells was used. The multiphysics simulations involved a region around the leading-edges which were simulated at the kinetic level; in the case shown in Fig. 1b, the kinetic-flow domain comprises 300 blocks with 575,000 cells. A reduced spatial extent with about half the blocks was also considered, corresponding to the 'thin' layer results in Fig. 2. Velocity space discretizations as $24^{3}$ and $32^{3}$ were used divided in $8 \times 4 \times 4$ or $8 \times 8 \times 4$ partitions. The flow demonstrates significant thermodynamic nonequilibrium in the kinetic-flow domain around the leading-edges as can be seen from the rotational temperature relaxation in Fig. 1c. The strong scaling for these cases are shown in Fig. 2, showing a 85 percent parallel efficiency from 2048 to 8192 processes.

For the coupling between the continuum solver and the kinetic solver, $\mathrm{M} \Phi \mathrm{C}$ employs state-based or flux-based hybrid techniques. In the state-based coupling (Fig. 3a), over an overlap region, the macroscopic variables are obtained from the microscopic solution while the latter is constructed from the macroscopic state. In the flux-based coupling, instead, at a designed interface, the numerical fluxes for one solver are obtained from the other as shown in Fig. $3 b$, while for hybrid NS-DSMC simulations, the choice of a state-based coupling is the preferable one due to the lower scattering error that it involves [12]. In the current deterministic DVM kinetic solver, the statistical scatter is absent, creating more flexibility in the used coupling technique. In the literature, 


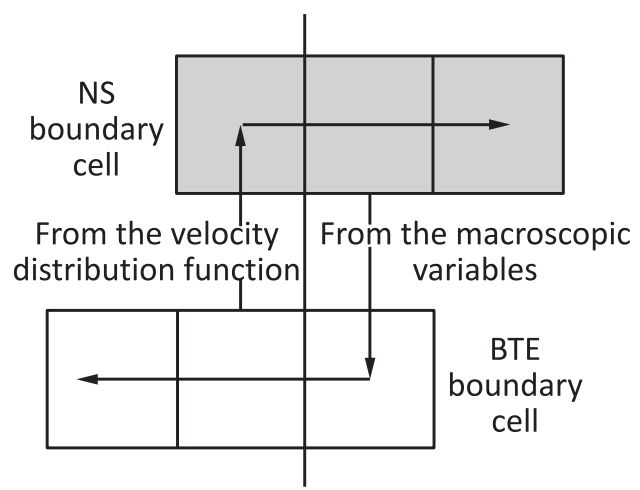

(a)

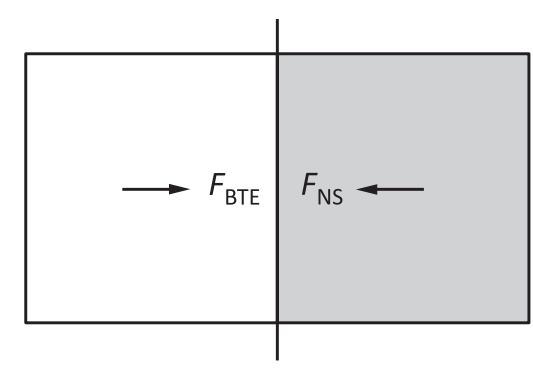

(b)

Figure 3 Coupling techniques, adapted from [12]: (a) state-based coupling; and (b) flux-based coupling

the domain decomposition is generally done during the simulation on the basis of a continuum breakdown parameter, for example, as done by other researchers in $[12,29]$. At the moment, this feature is still under development in $M \Phi C$ and the DVM domain has to be defined by the user in an input file. However, the framework can perform a reconfiguration of the different regions throughout the calculation when the domain definition file is modified by the user.

\section{FLOW MODELS AND NUMERICAL SCHEMES}

\subsection{Nondimensional Rykov Model}

Considering the flow of a diatomic gas, let us assume that the gas temperature is not too high, so that the vibrational degrees of freedom are not excited, and not too low, so that the rotational degrees of freedom can be considered to be fully excited. In this case, the particle distribution function $f(\mathbf{x}, \mathbf{c}, t, \zeta)$, which describes the state of the gas, will be a function not only of the spatial coordinate $\mathbf{x}$, the particle velocity $\mathbf{c}$, and the time $t$, but also of the rotational degrees of freedom $\zeta$. The Rykov model represents an extension of the Shakhov model where also rotational nonequilibrium is considered and has been proved to be a reliable kinetic approximation, up to the heat fluxes moments of the BTE, for this kind of flow $[7,18,30,31]$. Since the rotational degrees of freedom are considered fully excited, $\zeta$ is reduced by the model and the second distribution function is obtained. 
Employing the following nondimensional variables:

$$
\begin{aligned}
& \rho=\frac{\hat{\rho}}{\rho_{\infty}} ; \quad T=\frac{\hat{T}}{T_{\infty}} ; \quad u=\frac{\hat{c}}{\sqrt{2 R T_{\infty}}} ; \\
& t=\frac{\hat{t}}{\mu_{\infty} p_{\infty}^{-1}} ; \quad \mathbf{x}=\frac{\hat{\mathbf{x}}}{\sqrt{2 R T_{\infty}} \mu_{\infty} p_{\infty}^{-1}} ; \quad p=\frac{\hat{p}}{\rho_{\infty} R T_{\infty}} \\
& \mu=\frac{\hat{\mu}}{\mu_{\infty}} ; \quad q=\frac{\hat{q}}{\rho_{\infty}\left(2 R T_{\infty}\right)^{3 / 2}} ; \quad \tau=\frac{\hat{\tau}}{\mu_{\infty} p_{\infty}^{-1}},
\end{aligned}
$$

the nondimensional distribution functions of the model result

$$
F_{0}=\frac{\hat{F}_{0}}{\rho_{\infty}\left(2 R T_{\infty}\right)^{-3 / 2}} ; \quad F_{1}=\frac{\hat{F}_{1}}{m R T_{\infty} \rho_{\infty}\left(2 R T_{\infty}\right)^{-3 / 2}}
$$

thus, for the Rykov model written in terms of $F=m f$, one obtains

$$
\left.\begin{array}{c}
\frac{\partial F_{0}}{\partial t}+\mathbf{c} \frac{\partial F_{0}}{\partial \mathbf{x}}=\frac{F_{0}^{e q}-F_{0}}{\tau} ; \quad \frac{\partial F_{1}}{\partial t}+\mathbf{c} \frac{\partial F_{1}}{\partial \mathbf{x}}=\frac{\partial F_{1}^{e q}-F_{1}}{\partial \tau} ; \\
F_{0}^{\mathrm{eq}}=\frac{1}{Z_{r}} F_{0}^{r}+\left(1-\frac{1}{Z_{r}}\right) F_{0}^{t} ; \quad F_{1}^{\mathrm{eq}}=\frac{1}{Z_{r}} F_{1}^{r}+\left(1-\frac{1}{Z_{r}}\right) F_{1}^{t} ; \\
F_{0}^{r}=F_{M}(T)\left[1+\frac{8}{15} \omega_{0} \frac{q_{x}^{t}}{p} \frac{c^{\prime}{ }_{x}}{T}\left(\frac{\mathbf{c}^{\prime 2}}{T}-\frac{5}{2}\right)\right] ; \\
F_{0}^{t}=F_{M}\left(T_{t}\right)\left[1+\frac{8}{15} \frac{q_{x}^{t}}{p_{t}} \frac{c_{x}^{\prime}}{T_{t}}\left(\frac{\mathbf{c}^{\prime 2}}{T_{t}}-\frac{5}{2}\right)\right] ; \\
F_{1}^{t}=T F_{M}(T)\left[1+\frac{8}{15} \omega_{0} \frac{q_{x}^{t}}{p} \frac{c_{x}^{\prime}}{T}\left(\frac{\mathbf{c}^{\prime 2}}{T}-\frac{5}{2}\right)+4 \omega_{1}(1-\delta) \frac{q_{i}^{r} c_{i}^{\prime}}{p T}\right] ; \\
\left.F_{t}\right)\left[1+\frac{8}{15} \frac{q_{x}^{t}}{p_{t}} \frac{c_{x}^{\prime}}{T_{t}}\left(\frac{\mathbf{c}^{\prime 2}}{T_{t}}-\frac{5}{2}\right)+4(1-\delta) \frac{q_{i}^{r} c_{i}^{\prime}}{p_{t} T_{r}}\right]
\end{array}\right\}
$$

where

$$
F_{M}(T)=\frac{\rho}{(\pi T)^{3 / 2}} \exp \left(-\frac{\mathbf{c}^{\prime 2}}{T}\right)
$$

and the total collision time $\tau$ is expressed as $\mu_{t} / p_{t}$ with the viscosity determined from the translational temperature.

In a system of colliding particles, energy is transferred between the various internal modes. These collisions tend to drive the internal energy distributions towards their respective equilibrium state and the number of them necessary to 
push a particular mode to the equilibrium is the collision number, $Z$, associated to that mode [32]. The Rykov model is based on the assumption that the fraction of collisions involving the excitation of the rotational degrees of freedom, $Z_{r}$, is a given constant or a function of the flow temperatures. Several works provide an expression of $Z_{r}$ as a function of the temperature in the flow field. Probably, the first attempt to appear in the literature is the theoretical work of Parker [33] where, employing an empirical nonimpulsive model and assuming coplanar collisions and zero initial rotational energy, the following approximate expression is obtained:

$$
Z_{r}^{\mathrm{Par}}=\frac{\left(Z_{r}\right)_{\infty}}{1+\left(\pi^{3 / 2} / 2\right)\left(T^{*} / \hat{T}\right)^{1 / 2}+\left(\pi^{2} / 4+\pi\right)\left(T^{*} / \hat{T}\right)}
$$

where $T *=91.5 \mathrm{~K}$ is the characteristic temperature of the intermolecular potential and $\left(Z_{r}\right)_{\infty}=23.5$ is the limiting value suggested in [34]. While Parker's expression (2) is derived involving a large number of simplifying assumptions, the overall dependence on the temperature is in agreement with the more rigorous treatment of [35]. However, this expression does not involve any dependence on the different translational and rotational temperatures. Thus, in the recent literature, models derived from data fitting, either from numerical or experimental results, have been employed. In $[7,30,31]$, the following expression for the collision number is presented to be used with the Rykov model:

$$
Z_{r}^{\mathrm{Ryk}}=\frac{3}{4} \pi \frac{\psi(\tilde{T})}{\tilde{T}^{1 / 6}} \frac{9 \tilde{T}}{\tilde{T}+8} \frac{\hat{T}_{r}}{\hat{T}_{t}}\left[0.461+0.5581\left(\frac{\hat{T}_{t}}{1 K}\right)+0.0358\left(\frac{\hat{T}_{t}}{1 K}\right)^{2}\right]
$$

where

$$
\psi(\tilde{T})=0.767+0.233 \tilde{T}^{-1 / 6} \exp (-1.17[\tilde{T}-1]) ; \tilde{T}=\frac{\hat{T}_{t}}{T^{*}}
$$

An alternative expression for $Z_{r}\left(T_{t}, T_{r}\right)$ derived from MD simulations can be found in [36]:

$$
\begin{aligned}
& Z_{r}^{\mathrm{Val}} \\
& =\left[a_{1}\left(\frac{\hat{T}_{t}}{1 K}\right)^{1 / 4}+a_{2}\left(\frac{\hat{T}_{t}}{1 K}\right)^{-1 / 4}-a_{3}\left(\frac{\hat{T}_{t}}{1 K}-1000\right)\right]\left[1-b\left(1-\frac{\hat{T}_{r}}{\hat{T}_{t}}\right)\right]
\end{aligned}
$$

where $a_{1}=1.33868 ; a_{2}=-6.19992 ; a_{3}=-0.00107942$; and $0<b \leq 1$. It is important to notice that considering the moments of the Rykov model collision term, the relaxation process in the model is described as $\rho\left(T-T_{r}\right) /\left(Z_{r} \tau\right)$ while 
in $[33,36]$, Jeans equation is considered leading to $\rho\left(T_{t}-T_{r}\right) /\left(Z_{r} \tau\right)$. This means that the collision number in the Rykov model results in

$$
Z_{r}=0.6 Z_{r}^{\mathrm{Par} / \mathrm{Val}}
$$

For the viscosity law, Rykov and his coworkers [7,30,31] suggest

$$
\mu\left(T_{t}\right)=\mu\left(T^{*}\right) \frac{\tilde{T}^{2 / 3}}{\psi(\tilde{T})} ;
$$

otherwise, a simpler power law

$$
\mu\left(T_{t}\right)=\mu\left(T_{\infty}\right)\left(\frac{T_{t}}{T_{\infty}}\right)^{\omega}
$$

with an exponential factor of 0.72 [37] can be employed.

To make the system (1) complete, the values of the constants $\delta, \omega_{0}$, and $\omega_{1}$ need to be determined. In [38], $\omega_{0}=0.2354$ and $\omega_{1}=0.3049$ or $\omega_{0}=0.5$ and $\omega_{1}=0.286$ are given for diatomic gases. Both pairs of values have been successfully employed in $[30,31,38,39]$ with $\delta^{-1}=1.55$. In the present work, the values $\omega_{0}=0.5$ and $\omega_{1}=0.286$ are employed.

The dimensionless macroscopic quantities can be obtained from $F_{0}$ and $F_{1}$ by means of the following formulae:

$$
\begin{gathered}
\rho=\int_{-\infty}^{+\infty} F_{0} d \mathbf{c} ; \rho u_{x}=\int_{-\infty}^{+\infty} c_{x} F_{0} d \mathbf{c} ; \frac{3}{2} \rho T_{t}+\rho u_{x}^{2}=\int_{-\infty}^{+\infty} c^{2} F_{0} d \mathbf{c} ; \\
\rho T_{r}=\int_{-\infty}^{+\infty} F_{1} d \mathbf{c} ; \quad \frac{5}{2} T=\frac{3}{2} T_{t}+T_{r} ; \quad p_{t}=\rho T_{t} ; \quad p=\rho T ; \\
q_{x}^{t}=\int_{-\infty}^{+\infty} c_{x}^{\prime} \frac{\mathbf{c}^{\prime 2}}{2} F_{0} d \mathbf{c} ; \quad q_{x}^{r}=\int_{-\infty}^{+\infty} \frac{c^{\prime} x}{2} F_{1} d \mathbf{c} .
\end{gathered}
$$

\subsection{Gas-Kinetic Scheme for Near-Continuum Flows Based on the Rykov Model}

Integrating in time the nondimensional reduced Rykov model system (1) and taking the moments $\boldsymbol{\Psi}_{0}=\left(1, c_{x}, \mathbf{c}^{2}, 0\right)^{\mathrm{T}}$ of $\left.F_{0}\right|_{i} ^{n+1}$ and $\boldsymbol{\Psi}_{1}=(0,0,1,1)^{\mathrm{T}}$ of $\left.F_{1}\right|_{i} ^{n+1}$, the following can be obtained for the update of the nondimensional macroscopic variables: 


$$
\begin{aligned}
& \mathbf{W}_{i}^{n+1}=\mathbf{W}_{i}^{n}+\frac{1}{\Delta x} \sum_{m} \int_{t^{n}}^{t^{n+1}} \boldsymbol{\Psi}_{0}\left(\left.\left[\left.c_{x}\right|_{m} F_{0}\right]\right|_{i-1 / 2}-\left.\left[\left.c_{x}\right|_{m} F_{0}\right]\right|_{i+1 / 2}\right) d t \Delta c_{x} \\
& +\frac{1}{\Delta x} \sum_{m} \int_{t^{n}}^{t^{n+1}} \mathbf{\Psi}_{1}\left(\left.\left[\left.c_{x}\right|_{m} F_{1}\right]\right|_{i-1 / 2}-\left.\left[\left.c_{x}\right|_{m} F_{1}\right]\right|_{i+1 / 2}\right) d t \Delta c_{x}+\frac{\Delta t}{2}\left(\mathbf{S}_{i}^{n+1}+\mathbf{S}_{i}^{n}\right)
\end{aligned}
$$

where the source term is

$$
\frac{\Delta t}{2}\left(\mathbf{S}_{i}^{n+1}+\mathbf{S}_{i}^{n}\right)=\frac{\Delta t}{2}\left(0,0,0, \frac{\rho\left(\left.T\right|_{i} ^{n+1}-\left.T_{r}\right|_{i} ^{n+1}\right)}{Z_{r} \tau_{i}^{n+1}}+\frac{\rho\left(\left.T\right|_{i} ^{n}-\left.T_{r}\right|_{i} ^{n}\right)}{Z_{r} \tau_{i}^{n}}\right)^{\mathrm{T}}
$$

and the velocity (phase) space is discretized using a uniformly spaced method with the trapezoidal rule for the evaluation of the moments of the distribution functions. As previously shown in $[40,41]$, reconstructing the time-dependent distribution functions at the cell-faces, i. e., $\left.F_{0}\right|_{i \pm 1 / 2}$ and $\left.F_{1}\right|_{i \pm 1 / 2}$, consistently on the basis of the CE solution of the Rykov model:

$$
\left.\begin{array}{l}
F_{0}=F_{0}^{\mathrm{eq}}-\tau\left[\frac{\partial F_{M}\left(T_{t}\right)}{\partial t}+\mathbf{c} \frac{\partial F_{M}\left(T_{t}\right)}{\partial \mathbf{x}}\right] ; \\
F_{1}=F_{1}^{\mathrm{eq}}-\tau\left[\frac{\partial\left(T_{r} F_{M}\left(T_{t}\right)\right)}{\partial t}+\mathbf{c} \frac{\partial\left(T_{r} F_{M}\left(T_{t}\right)\right)}{\partial \mathbf{x}}\right]
\end{array}\right\}
$$

for a well-resolved flow, it is possible to obtain:

$$
\begin{aligned}
& F_{0}=F_{0}^{\mathrm{eq}}-\tau\left(\frac{\partial F_{M}\left(T_{t}\right)}{\partial t}+c_{x} \frac{\partial F_{M}\left(T_{t}\right)}{\partial x}\right)+t \frac{\partial F_{M}\left(T_{t}\right)}{\partial t} \\
& F_{1}=F_{1}^{\mathrm{eq}}-\tau\left(\frac{\partial\left(T_{r} F_{M}\left(T_{t}\right)\right)}{\partial t}+c_{x} \frac{\partial\left(T_{r} F_{M}\left(T_{t}\right)\right)}{\partial x}\right)+t \frac{\partial\left(T_{r} F_{M}\left(T_{t}\right)\right)}{\partial t}
\end{aligned}
$$

where the derivatives are obtained analytically with the derivative of the Maxwellian defined as follows:

$$
\begin{gathered}
\frac{\partial F_{M}}{\partial \alpha}=F_{M}\left[\frac{1}{\rho} \frac{\partial \rho}{\partial \alpha}+\frac{1}{T}\left(\frac{\mathbf{c}^{\prime 2}}{T}-\frac{3}{2}\right) \frac{\partial T}{\partial \alpha}+2 \frac{c_{x}^{\prime}}{T} \frac{\partial u_{x}}{\partial \alpha}\right] ; \\
F_{0}^{\mathrm{eq}}=F_{0}^{t}+\frac{F_{0}^{r}-F_{0}^{t}}{Z_{r}} ; \quad F_{1}^{\mathrm{eq}}=F_{1}^{t}+\frac{F_{1}^{r}-F_{1}^{t}}{Z_{r}} .
\end{gathered}
$$

The time derivatives of the macroscopic variables can be obtained in terms of the space derivatives by means of the compatibility condition for the Rykov model:

$$
\begin{array}{r}
\int_{-\infty}^{+\infty}\left[\boldsymbol{\Psi}_{0}\left(\frac{\partial F_{M}\left(T_{t}\right)}{\partial t}+c_{x} \frac{\partial F_{M}\left(T_{t}\right)}{\partial x}\right)+\boldsymbol{\Psi}_{1}\left(\frac{\partial T_{r} F_{M}\left(T_{t}\right)}{\partial t}+c_{x} \frac{\partial T_{r} F_{M}\left(T_{t}\right)}{\partial x}\right)\right] d \mathbf{c} \\
=\mathbf{S}
\end{array}
$$


then,

$$
\begin{aligned}
\int_{-\infty}^{+\infty}\left[\boldsymbol{\Psi}_{0} \frac{\partial F_{M}\left(T_{t}\right)}{\partial t}+\right. & \left.\boldsymbol{\Psi}_{1} \frac{\partial T_{r} F_{M}\left(T_{t}\right)}{\partial t}\right] d \mathbf{c} \\
& =\mathbf{S}-\int_{-\infty}^{+\infty}\left(\boldsymbol{\Psi}_{0} c_{x} \frac{\partial F_{M}\left(T_{t}\right)}{\partial x}+\boldsymbol{\Psi}_{1} c_{x} \frac{\partial T_{r} F_{M}\left(T_{t}\right)}{\partial x}\right) d \mathbf{c}
\end{aligned}
$$

which, as explained in $[40,41]$, leads to

$$
\frac{\partial \mathbf{W}_{\mathbf{R}}}{\partial t}=\mathbf{S}-\frac{\partial \mathbf{Q}_{\mathbf{R}}}{\partial x}
$$

where

$$
\mathbf{W}_{R}=\left(\rho, \rho u_{x}, \frac{5}{2} \rho T+\rho u_{x}^{2}, \rho T_{r}\right)^{\mathrm{T}} ; \mathbf{Q}_{R}=\left[\begin{array}{c}
\rho u_{x} \\
\frac{1}{2} \rho T_{t}+\rho u_{x}^{2} \\
\frac{5}{2} \rho T_{t} u_{x}+\rho u_{x}^{3}+\rho T_{r} u_{x} \\
\rho T_{r} u_{x}
\end{array}\right]
$$

Introducing the CE solutions (7) of the Rykov model (1) with a modified collision time $\tau^{*}$ :

$$
\begin{aligned}
& F_{0}=F_{0}^{\mathrm{eq}}-\tau^{*}\left(\frac{\partial F_{M}\left(T_{t}\right)}{\partial t}+\mathbf{c} \frac{\partial F_{M}\left(T_{t}\right)}{\partial \mathbf{x}}\right) \\
& F_{1}=F_{1}^{\mathrm{eq}}-\tau^{*}\left(\frac{\partial\left(T_{r} F_{M}\left(T_{t}\right)\right)}{\partial t}+c_{x} \frac{\partial\left(T_{r} F_{M}\left(T_{t}\right)\right)}{\partial x}\right)
\end{aligned}
$$

back in the model equation, it is possible to obtain

$$
\left.\begin{array}{r}
\tau^{*} D F_{M}\left(T_{t}\right)+\tau^{*} \tau D^{2} F_{M}\left(T_{t}\right)=\tau D F_{0}^{\mathrm{eq}} ; \\
\tau^{*} D\left(T_{r} F_{M}\left(T_{t}\right)\right)+\tau^{*} \tau D^{2}\left(T_{r} F_{M}\left(T_{t}\right)\right)=\tau D F_{1}^{\mathrm{eq}} .
\end{array}\right\}
$$

Since the difference in the relaxation rate between translational and rotational processes is inherited in the collision number, $Z_{r}$, let us define a single modified collision time by taking the moment relative to the total heat flux. Thus, multiplying the first of Eqs. (10) by $c^{\prime}{ }_{x} c^{\prime 2}$ and the second one by $c^{\prime}{ }_{x}$ and adding the two resulting equations, one obtains:

$$
\tau^{*}=\frac{\tau\left(\left\langle D F_{0}^{\mathrm{eq}}\right\rangle+\left\langle D F_{1}^{\mathrm{eq}}\right\rangle\right)}{\left(\left\langle D F_{M}\left(T_{t}\right)\right\rangle+\left\langle D\left\{T_{r} F_{M}\left(T_{t}\right)\right\}\right\rangle\right)+\tau\left(\left\langle D^{2} F_{M}\left(T_{t}\right)\right\rangle+\left\langle D^{2}\left\{T_{r} F_{M}\left(T_{t}\right)\right\}\right\rangle\right)} .
$$


Let us simplify the numerical evaluation of this closure of the CE expansion by neglecting the terms relative to the Prandtl number correction introduced in the Rykov model; then,

$$
\tau^{*}=\tau\left(1+\tau \frac{\left\langle D^{2} F_{M}\left(T_{t}\right)\right\rangle+\left\langle D^{2}\left\{T_{r} F_{M}\left(T_{t}\right)\right\}\right\rangle}{\left\langle D F_{M}\left(T_{t}\right)\right\rangle+\left\langle D\left\{T_{r} F_{M}\left(T_{t}\right)\right\}\right\rangle}\right)^{-1}
$$

where

$$
\left.\begin{array}{c}
\left\langle D F_{M}\left(T_{t}\right)\right\rangle=\frac{5}{4} \frac{\partial\left(\rho T_{t}^{2}\right)}{\partial x}+\frac{5}{2} \rho T_{t}\left(\frac{\partial u_{x}}{\partial t}+u_{x} \frac{\partial u_{x}}{\partial x}\right) ; \\
\left\langle D^{2} F_{M}\left(T_{t}\right)\right\rangle=\frac{5}{2}\left(\frac{\partial^{2}\left(\rho T_{t}^{2}\right)}{\partial t \partial x}+\frac{\partial^{2}\left(\rho T_{t}^{2} u_{x}\right)}{\partial x^{2}}\right) \\
+T_{t}\left(5 \frac{\partial \rho}{\partial t} \frac{\partial u_{x}}{\partial t}+\frac{5}{2} u_{x}\left(\frac{\partial \rho}{\partial t} \frac{\partial u_{x}}{\partial x}+\frac{\partial \rho}{\partial x} \frac{\partial u_{x}}{\partial t}\right)+\left(\frac{11}{2} T_{t}+5 u_{x}^{2}\right) \frac{\partial \rho}{\partial x} \frac{\partial u_{x}}{\partial x}\right) \\
+\rho\left(5 \frac{\partial T_{t}}{\partial t} \frac{\partial u_{x}}{\partial t}+\frac{5}{2} u_{x}\left(\frac{\partial T_{t}}{\partial t} \frac{\partial u_{x}}{\partial x}+\frac{\partial T_{t}}{\partial x} \frac{\partial u_{x}}{\partial t}\right)+\left(11 T_{t}+5 u_{x}^{2}\right) \frac{\partial T_{t}}{\partial x} \frac{\partial u_{x}}{\partial x}\right) \\
+\rho T_{t}\left(\frac{5}{2}\left(\frac{\partial u_{x}}{\partial t}\right)^{2}+\frac{5}{2} u_{x} \frac{\partial^{2} u_{x}}{\partial t \partial x}+8 \frac{\partial u_{x}}{\partial t} \frac{\partial u_{x}}{\partial x}+\left(\frac{11}{4} T_{t}+\frac{5}{2} u_{x}^{2}\right) \frac{\partial^{2} u_{x}}{\partial^{2} x}\right. \\
\left.+\rho\left\{T_{r} F_{M}\left(T_{t}\right)\right\}\right\rangle=\frac{1}{2} \frac{\partial}{\left(\rho T_{r} T_{t}\right)} \partial x+\rho u_{x}\left(\frac{\partial u_{x}}{\partial x}\right) \\
+\rho T_{r}\left(\frac{\partial^{2} u_{x}}{\partial^{2} t}+2 u_{x} \frac{\partial^{2} u_{x}}{\partial t \partial x}+4 \frac{\partial u_{x}}{\partial t} \frac{\partial u_{x}}{\partial x}+\left(\frac{T_{t}}{2}+u_{x}^{2} \frac{\partial u_{x}}{\partial x}\right) ;\right. \\
\left.+D^{2}\left\{T_{r} F_{M}\left(T_{t}\right)\right\}\right\rangle=\frac{\partial^{2}\left(\rho T_{r} T_{t}\right)}{\partial t \partial x}+\frac{\partial^{2}\left(\rho T_{r} T_{t} u_{x}\right)}{x^{2}} \\
+T_{r}\left(2 \frac{\partial \rho}{\partial t} \frac{\partial u_{x}}{\partial t}+2 u_{x} \frac{\partial \rho}{\partial t} \frac{\partial u_{x}}{\partial x}+2 u_{x} \frac{\partial \rho}{\partial x} \frac{\partial u_{x}}{\partial t}+\left(T_{t}+2 u_{x}^{2}\right) \frac{\partial \rho}{\partial x} \frac{\partial u_{x}}{\partial x}\right) \\
+\rho T_{r} \frac{\partial T_{t}}{\partial x} \frac{\partial T_{r}}{\partial x} \\
\left.+2 u_{x} \frac{\partial u_{x}}{\partial x}+2 u_{x} \frac{\partial T_{r}}{\partial x} \frac{\partial u_{x}}{\partial t}+\left(T_{t}+2 u_{x}^{2}\right) \frac{\partial \rho}{\partial x}\right)
\end{array}\right\}
$$

The second derivatives $\partial^{2} /(\partial t \partial x)$ can be expressed in terms of only spatial derivatives thanks to the compatibility conditions (8) 


$$
\frac{\partial^{2} \mathbf{W}_{R}}{\partial t \partial x}=\frac{\partial \mathbf{S}}{\partial x}-\frac{\partial^{2} \mathbf{Q}_{R}}{\partial x^{2}}
$$

where $\mathbf{W}_{R}$ and $\mathbf{Q}_{R}$ are defined in Eqs. (9), while the second time derivative of the mean velocity can be obtained considering the second-order compatibility conditions for the translational part:

$$
\left.\begin{array}{rl}
\frac{\partial^{2} \mathbf{W}_{S}}{\partial t \partial x}=-\frac{\partial^{2} \mathbf{Q}_{S}}{\partial x^{2}} ; & \\
\frac{\partial^{2} \mathbf{W}_{S}}{\partial^{2} t}=-2 \int_{-\infty}^{+\infty} \boldsymbol{\Psi} c_{x} \frac{\partial^{2} F_{M}(T)}{\partial t \partial x} d \mathbf{c} \\
-\int_{-\infty}^{+\infty} \mathbf{\Psi} c_{x}^{2} \frac{\partial^{2} F_{M}(T)}{\partial^{2} x} d \mathbf{c}=-\frac{\partial^{2} \mathbf{Q}_{S}^{\prime}}{\partial t \partial x}-\frac{\partial^{2} \mathbf{Q}^{\prime \prime}{ }_{S}}{\partial x^{2}}
\end{array}\right\}
$$

where

$$
\begin{gathered}
\mathbf{W}_{S}=\left(\rho, \rho u_{x}, \frac{3}{2} \rho T_{t} u_{x}+\rho u_{x}^{2}\right)^{\mathrm{T}} ; \\
\left.\mathbf{Q}_{S}=\left(\rho u_{x}, \frac{1}{2} \rho T_{t}+\rho u_{x}^{2}, \frac{5}{2} \rho T_{t} u_{x}+\rho u_{x}^{3}\right)^{\mathrm{T}} \cdot\right\} \\
\mathbf{Q}^{\prime}{ }_{S}=\left(2 \rho u_{x}, \quad \rho T_{t}+2 \rho u_{x}^{2}\right)^{\mathrm{T}} ; \mathbf{Q}^{\prime \prime}{ }_{S}=\left(\frac{1}{2} \rho T_{t}+\rho u_{x}^{2}, \frac{2}{2} \rho T_{t} u_{x}+\rho u_{x}^{3}\right)^{\mathrm{T}} .
\end{gathered}
$$

as discussed in $[40,41]$.

Finally, similar to $[20,21]$, since $\left\langle D F_{M}(T)\right\rangle$ and $\left\langle D^{2} F_{M}(T)\right\rangle$ will be sensitive to numerical errors (especially, close to equilibrium regions where they tend to vanish, and to impose that the physical stress inside the shock layer should be larger than the stress in the NS equation), a limiter is needed. In the current work, the following nonlinear limiter is used:

$$
\begin{aligned}
\tau^{*}=\tau(1+\max (-0.5, \min & (-0.5(1.0-\exp (-c \times \mathrm{Kn})), \\
\tau & \left.\left.\left.\frac{\left\langle D^{2} F_{M}\left(T_{t}\right)\right\rangle+\left\langle D^{2}\left\{T_{r} F_{M}\left(T_{t}\right)\right\}\right\rangle}{\left\langle D F_{M}\left(T_{t}\right)\right\rangle+\left\langle D\left\{T_{r} F_{M}\left(T_{t}\right)\right\}\right\rangle}\right)\right)\right)^{-1} .
\end{aligned}
$$

where $\mathrm{Kn}$ is the local Knudsen number based on the gradients of the macroscopic variables defined as in $[12,42]$

$$
\mathrm{Kn}=\lambda \max \left(\frac{|\triangle \rho|}{\rho}, \frac{\left|\triangle u_{x}\right|}{u_{x}}, \frac{\left|\triangle T_{t}\right|}{T_{t}}, \frac{\left|\triangle T_{r}\right|}{T_{r}}, \frac{\left|T_{t}-T_{r}\right|}{2 T_{r}}\right)
$$

and the function $f(\mathrm{Kn})=-0.5(1.0+\exp (-c \mathrm{Kn}))$ is used to obtain a smoother transition from $\tau^{*}=\tau$ to $\tau^{*}=2 \tau$. Here, the parameter $c=4.01341$ is calculated in order to have at least a starting $\tau^{*}=1.1 \tau$ at $\mathrm{Kn}=0.05$. 


\subsection{Diffuse Wall Boundary Conditions}

The gas evolution at a solid boundary is modeled assuming that particles hit the wall with a distribution function according to the flow conditions whereas they are reflected with:

- a Maxwellian distribution according to the wall state for fully accommodation boundary (viscous wall);

- the same distribution function for specular reflection boundary (inviscid wall); and

- a combination of diffuse and specular boundaries depending on the accommodation coefficient $\sigma$.

Therefore, the final gas distribution function at the wall can be written as

$$
F=\sigma F_{M u>0}+F_{u<0}+(1-\sigma) F_{u>0}
$$

where $u<0$ and $u>0$ represent the velocities of particles hitting the wall and reflected by the wall, respectively, while $F_{M}$ and $F$ are the Maxwellian and the nonequilibrium distribution functions at the wall. The fluid state at the wall can be extrapolated from the domain. In the present work, fully accommodated walls have been employed, i. e., $\sigma=1$.

\section{RESULTS AND DISCUSSION}

\subsection{Assessment of the Gas-Kinetic Scheme}

Computational and memory cost is a major drawback for DVM in hybrid simulations and the use of the GKS to reduce the extension of the domain where

Table 1 Test cases details

\begin{tabular}{|c|c|c|c|c|c|c|}
\hline $\begin{array}{l}\text { Test } \\
\text { case }\end{array}$ & M & $\lambda_{\infty}$ & $\begin{array}{c}T_{\infty} \\
\mathrm{K}\end{array}$ & $\begin{array}{c}T_{\text {wall }}, \\
\mathrm{K}\end{array}$ & $Z_{r}$ & Solver \\
\hline Shock & 2.8 & & & & $\begin{array}{ll}4.2 & \text { Eq. (6) }\end{array}$ & $\overline{\mathrm{KS}} / \mathrm{GKS} \tau * / \mathrm{DVM}$ \\
\hline Shock & 10 & & & & $5.0 \quad$ Eq. (6) & $\mathrm{KS} / \mathrm{GKS} \tau * / \mathrm{DVM}$ \\
\hline Plate & 4.89 & $1.2 \mathrm{~mm}$ & 116 & 290 & Eq. (3) Eq. (5) & GKS/DVM \\
\hline Wedge & 4 & $0.1 \mathrm{~mm}$ & 185.6 & 293.3 & Eq. (3) Eq. (5) & GKS/DVM \\
\hline Cylinder & 12 & $\begin{array}{c}\text { Diameter } / 100 ; \\
\text { Diameter }=80 \mathrm{~mm}\end{array}$ & 217.5 & 1000 & Eq. (3) Eq. (5) & GKS/Hybrid \\
\hline Waverider & 8 & $\begin{array}{l}\text { Length } /\left(2.5 \cdot 10^{3}\right) \text {, } \\
\text { Diameter }_{\mathrm{LE}} / 2.5\end{array}$ & 116 & $2.5 T$ & Eq. (4) Eq. (6) & GKS \\
\hline
\end{tabular}


it is strictly required represents a preferable alternative. To support this assertion, different test cases for a wide range of Mach numbers have been considered. Details of the simulations are reported in Table 1 and wall boundary conditions are considered fully accommodated. As shown in Figs. $4 a$ and $5 a$, in contrast to traditional single-/multitemperature NS approaches $[44,45]$, the GKS is able to resolve shock structures with and without rotational nonequilibrium. However, due to the continuum formulation, it predicts steeper shocks relative to

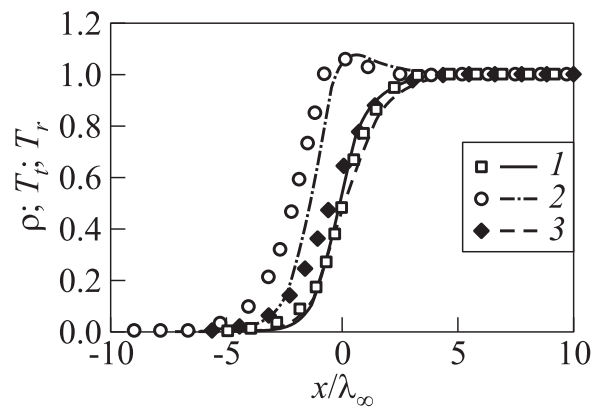

(a)

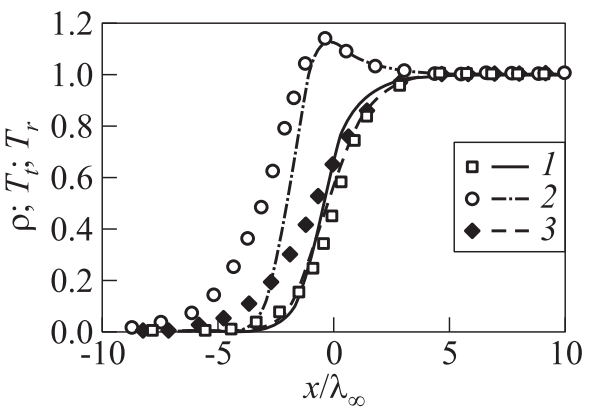

(b)

Figure 4 Nondimensional $\rho(1), T_{t}(2)$, and $T_{r}$ (3) normalized profiles, i.e., $(\phi$ $\left.-\phi_{-\infty}\right) /\left(\phi_{\infty}-\phi_{-\infty}\right)$, for normal shocks in nitrogen. Velocity space cells size is equal to $0.5 u_{\infty}$. The DSMC results (signs) are from [43]; curves refer to GKS based on Rykov model; viscosity power law with exponential 0.72 : (a) $\mathrm{M}=2.8$ and $Z_{r}=4.2$; and $(b) \mathrm{M}=10$ and $Z_{r}=5$

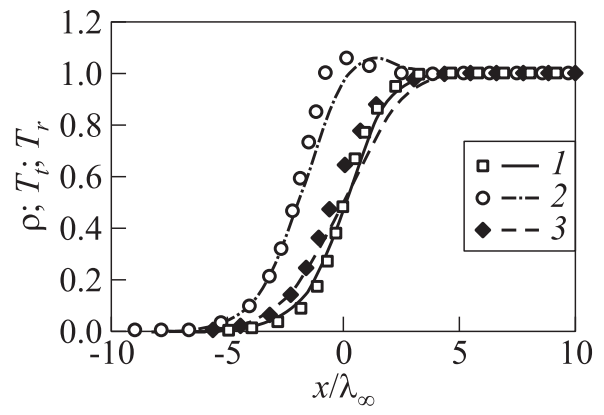

(a)

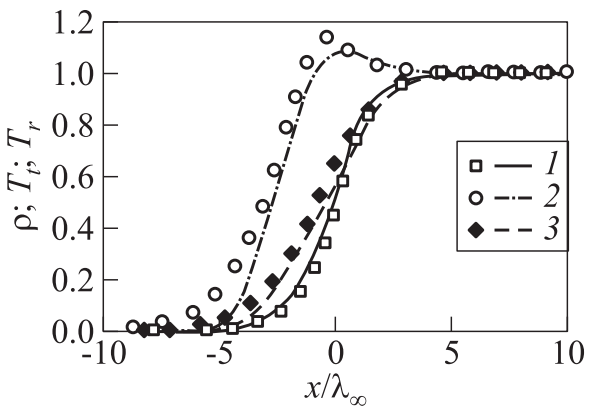

(b)

Figure 5 Nondimensional $\rho(1), T_{t}(2)$, and $T_{r}$ (3) normalized profiles, i.e., $(\phi$ $\left.-\phi_{-\infty}\right) /\left(\phi_{\infty}-\phi_{-\infty}\right)$, for normal shocks in nitrogen. Modified collision time $\tau^{*}$ is defined by Eqs. (11)-(19) and velocity space cells size is equal to $0.5 u_{\infty}$. The DSMC results are from [43]; curves refer to GKS $\tau^{*}$ based on Rykov model; viscosity power law with exponential 0.72: (a) $\mathrm{M}=2.8$ and $Z_{r}=4.2$; and $(b) \mathrm{M}=10$ and $Z_{r}=5$ 


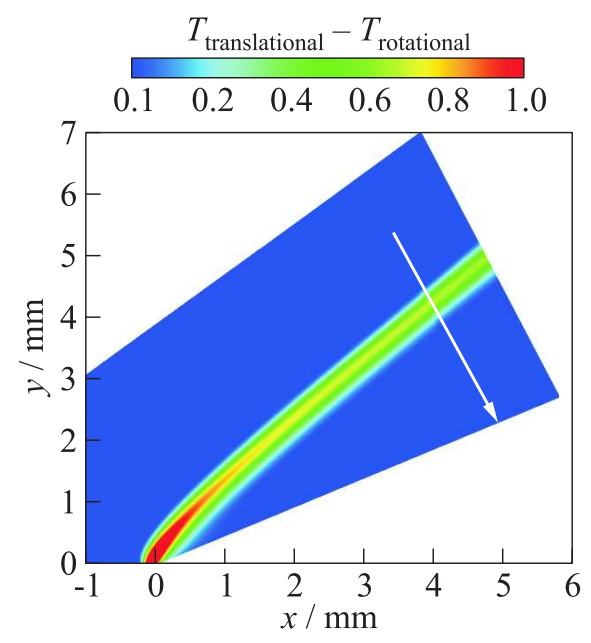

Figure 6 Mach 4 nitrogen flow around a 25 degree wedge; velocity space cells size is equal to $0.5 u_{\infty}$ : temperatures difference, GKS solution

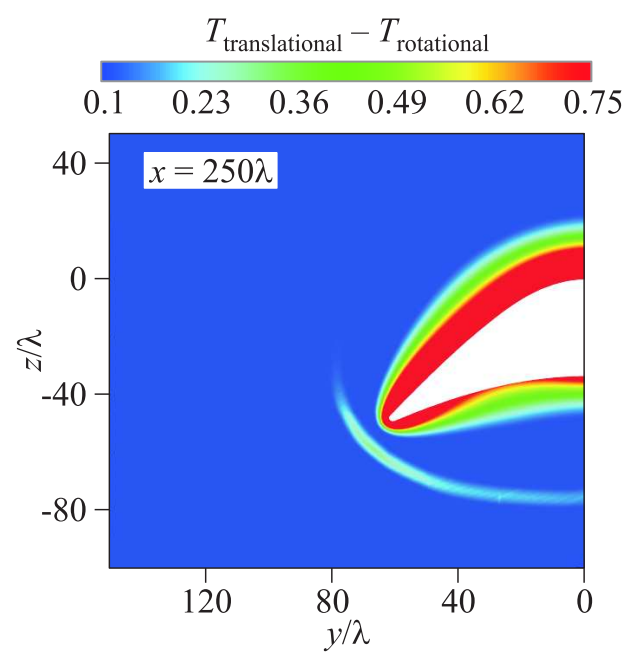

Figure 7 Mach 8 waverider, GKS solution: temperatures difference

DSMC solutions. Shock-structure predictions in better agreement with DSMC results can be obtained when Eq. (11) is employed to modify the collision time as shown in Fig. $5 a$. On the other hand, employing the modified collision time slightly affects the accuracy in the subsonic region of the shock. The main differences between GKS and DSMC results can be observed for the higher Mach number cases in Figs. $4 b$ and $5 b$. This is probably due to the use of the CE expansion which does not allow to represent the typical bi-modal behavior of the distribution function across shock waves at very high Mach numbers $(>4)$.

The capability of the GKS to resolve multiple temperatures across shock structures, as shown also in Figs. 6 and 7 for the wedge and waverider cases, respectively, makes possible the reduction of the domain where more complex approaches, such as the DVM, are necessary. Indeed, as it is possible to observe from Figs. 8-11, the GKS results prove to be in good agreement with DVM and DSMC calculations with the biggest differences between GKS and DVM, defined here as

$$
\Delta=\frac{\left|\phi_{\mathrm{DVM}}-\phi_{\mathrm{GKS}}\right|}{\left|\phi_{\mathrm{DVM}}\right|} \cdot 100 \%,
$$

occurring when the local Knudsen number (see Eq. (19)) is much higher than the commonly employed threshold, i. e., 0.05 [47], for the continuum breakdown. Furthermore, in both two-dimensional cases, the quantities at the wall are pre- 


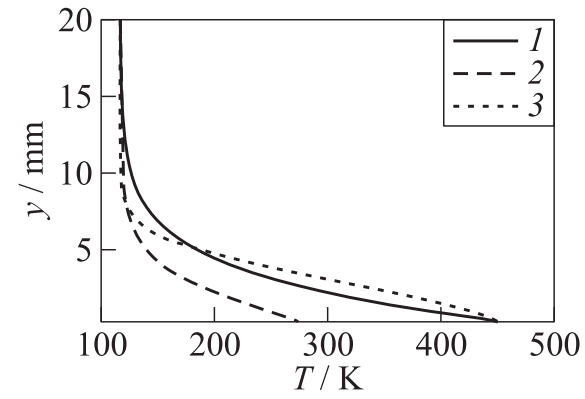

(a)

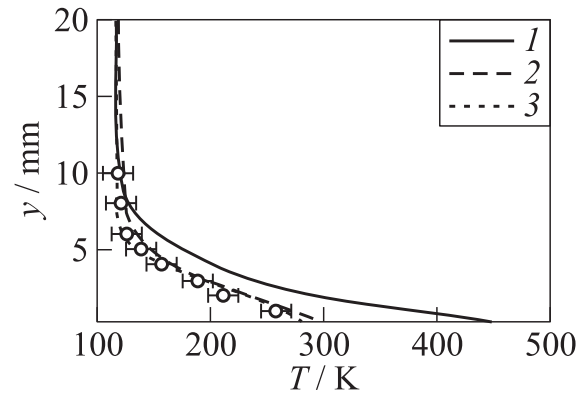

(b)

Figure 8 Mach 4.89 nitrogen flow around a flat plate; velocity space cells size is equal to $0.5 u_{\infty}$ : (a) $T_{t}$; (b) $T_{r} ; 1-$ DVM; 2 - DSMC; and 3 - GKS based on Rykov model. The DSMC calculations (2) and experimental data (signs) are reported in [46]; $\mathrm{Kn}_{x}=\lambda_{\infty} / x=0.24 ; x=5 \mathrm{~mm}$

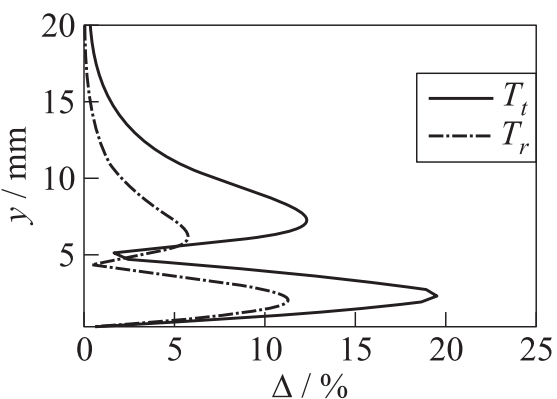

(a)

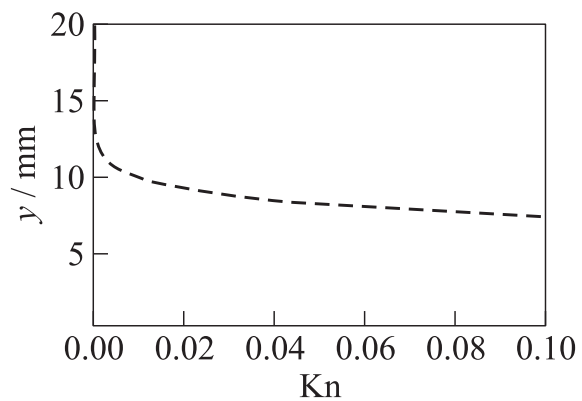

(b)

Figure 9 The GKS and DVM results difference (a) and local Kn (see Eq. (19)) (b). Mach 4.89 nitrogen flow around a flat plate; velocity space cells size is equal to $0.5 u_{\infty}$

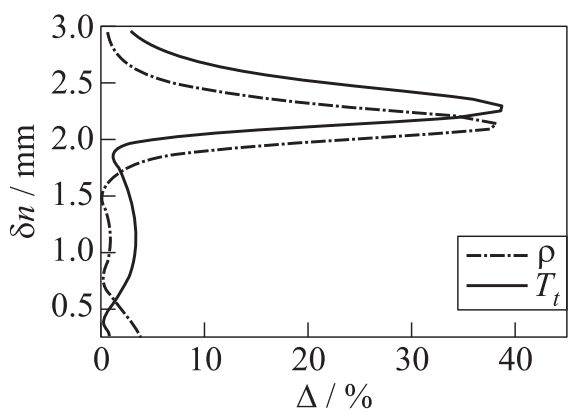

(a)

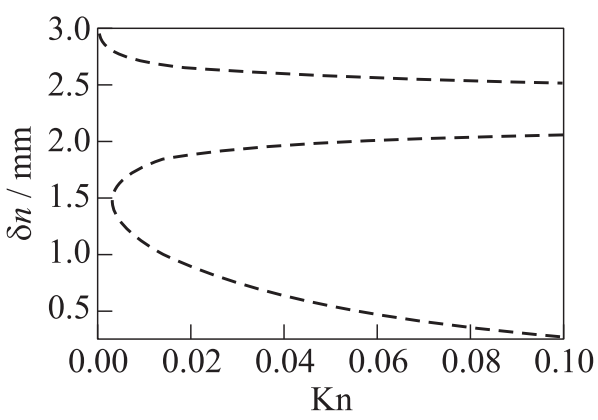

(b)

Figure 10 Mach 4 nitrogen flow around a 25 degree wedge; velocity space cells size is equal to $0.5 u_{\infty}$ : GKS and DVM results difference $(a)$ and local Kn (see Eq. (19)) (b) 


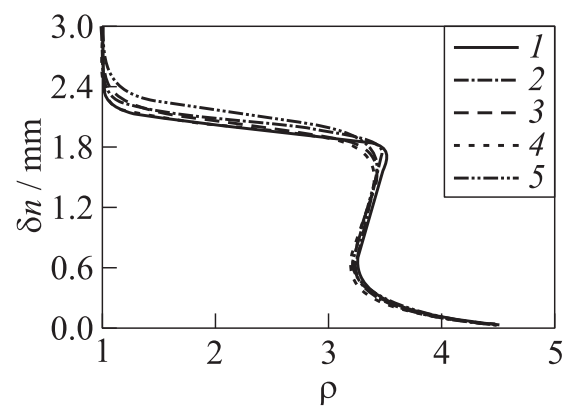

(a)

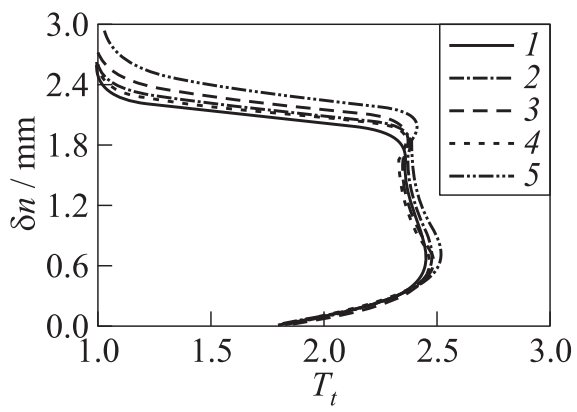

(b)

Figure 11 Mach 4 nitrogen flow around a 25 degree wedge: 1 - CFD; 2 - hybrid; 3 - DSMC; 4 - GKS based on Rykov model; and 5-DVM based on Rykov model. Velocity space cells size is equal to $0.5 u_{\infty}$. The DSMC, hybrid approach, and continuum results reported are from [11]

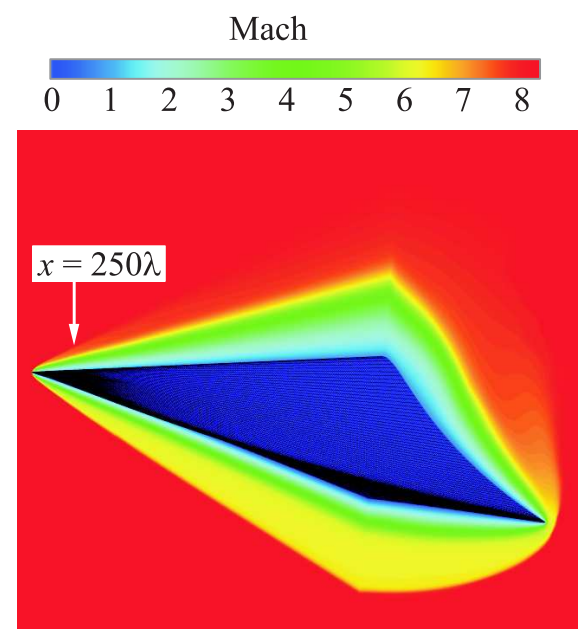

Figure 12 Mach 8 waverider, GKS solution: Mach contours. (Refer color plate, p. XXVIII.) dicted with a difference less than $5 \%$ relative to DVM calculations and Fig. $11 b$ also shows a good agreement between GKS and hybryd method results. Regarding the waverider case, as expected, the GKS predicts a flow field with high thermal nonequilibrium around the waverider nose where also important viscous interaction effects occur and in the boundary layer over the upper body surface (Figs. 7 and 12).

For the cylinder test case, Fig. 13 shows density and temperatures profiles for the section at 45 degree respective to the symmetry plane. The GKS correctly predicts the shock position as well as quantities and gradients at the wall in comparison to the results of the Modular Particle-

Continuum (MPC) method of [42]; however, a thinner shock is predicted. A better shock structure prediction can be obtained using the hybrid approach as can be observed in Fig. 14. Here, for the hybrid approach, the DVM is employed in a region around the bow shock while the rest of the domain is simulated using the GKS (Fig. 15). The information exchange between the two solvers has 


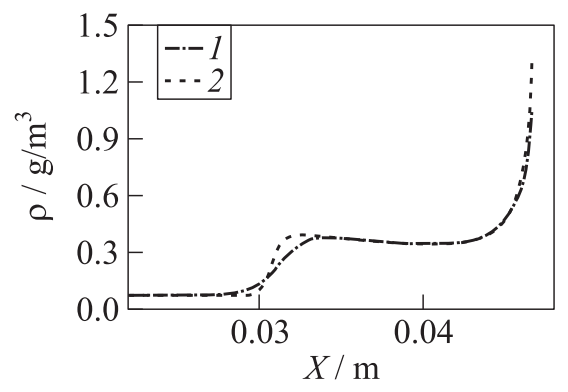

(a)

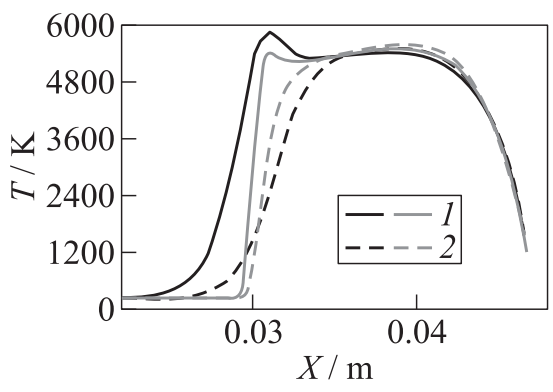

(b)

Figure 13 The $\rho(a)$ and $T_{t}$ (solid curves) and $T_{r}$ (dashed curves) profiles $(b)$ at $45^{\circ}$. Mach 12 nitrogen flow around a cylinder; velocity space cells size is equal to $0.5 u_{\infty}$. The MPC results (1) are from [42]; curves 2 refer to GKS based on Rykov model

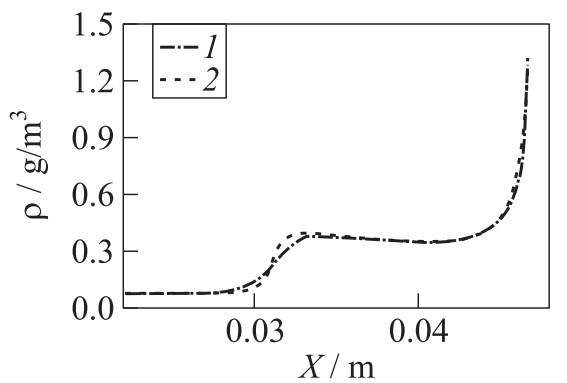

(a)

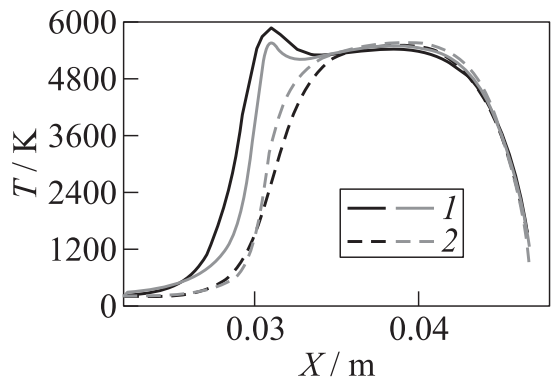

(b)

Figure 14 The $\rho(a)$ and $T_{t}$ (solid curves) and $T_{r}$ (dashed curves) profiles $(b)$ at $45^{\circ}$. Mach 12 nitrogen flow around a cylinder; velocity space cells size is equal to $0.5 u_{\infty}$. The MPC results (1) are from [42]; curves 2 refer to GKS based on Rykov model / DVM

been handled employing the state-based coupling (see Fig. $3 a$ ), described in section 2 with an overlap region extension of about 10 cells. This means that across the overlap region, the distribution function employed as boundary condition for the DVM is reconstructed using the CE expansion (see Eq. (7)), where the macroscopic variables and the relative derivatives are defined by the GKS solutions; vice versa: the macroscopic variables and the relative derivatives needed for the GKS fluxes are obtained from the DVM solution. The differences can be still observed between the hybrid results from the present work and the ones in [42]. The reason of these discrepancies can be found in the different methods employed. Indeed, in [42], a DSMC method is used where rarefaction effect are dominant while in the present work, a DVM, which solves only a simplified model of the BTE where the collision time $\tau$ does not depend on the particle 


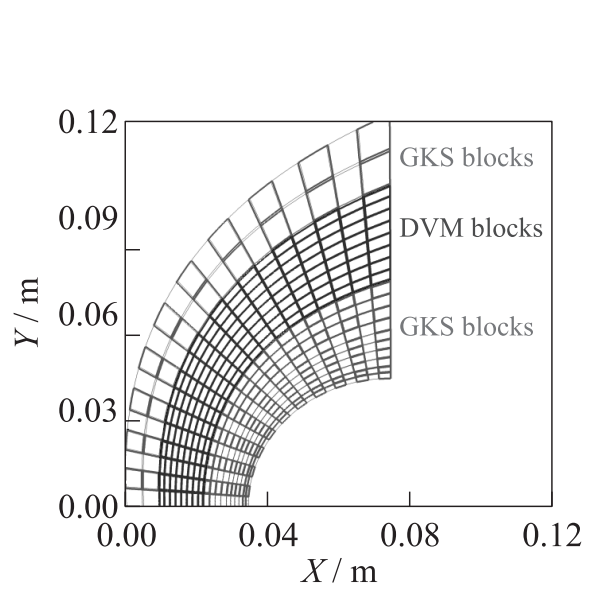

Figure 15 The DVM and GKS domains. Mach 12 nitrogen flow around a cylinder; velocity space cells size is equal to $0.5 u_{\infty}$

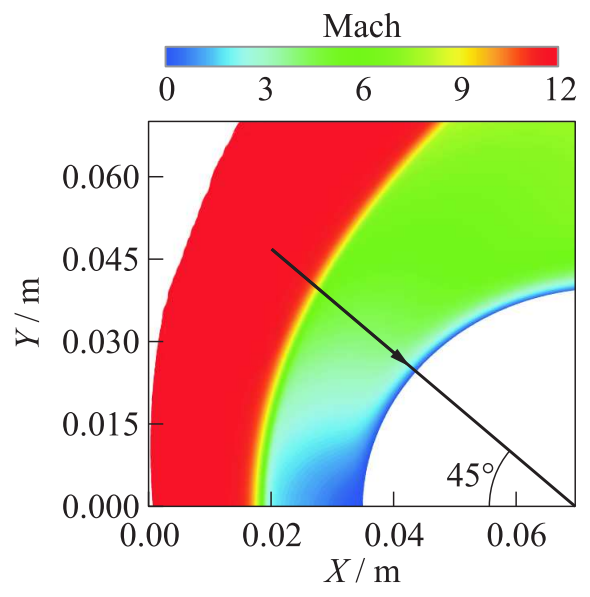

Figure 16 The Mach contours. Mach 12 nitrogen flow around a cylinder; velocity space cells size is equal to $0.5 u_{\infty}$

velocities, is employed. When employed in a hybrid simulation, as it is possible to notice from Fig. 16, the transition between the GKS and the DVM solvers at the interface is naturally smooth, no blending of the two solutions has been applied in the overlap region, due to the common root of the approaches. This and the extended validity of the the GKS also suggest a reduction of the hybrid simulations' sensitivity to the positioning of the interfaces. However, the latter point requires further investigations.

\subsection{Computational and Memory Cost}

The GKS is implemented so that the particle velocity space dimensions depend on the local state, while due its complexity, this feature is not available in the DVM for the kinetic Boltzmann equations in M $\Phi \mathrm{C}$. To perform a fair comparison of the computational time for the two approaches, constant velocity spaces were used. The runs have been performed on Intel ${ }^{\circledR}$ Xeon ${ }^{\circledR}$ processors at the University of Liverpool cluster "Chadwick" and solutions have been considered converged when the $L^{2}$-norm of the update between two consecutive solutions is lower than $10^{-7}$ for the normal shock cases and $10^{-8}$ for the two-dimensional cases. Furthermore, it needs to be reminded that the halo exchange in the GKS involves only the flow state while the DVM needs to exchange the full velocity space and this represents an advantage of the GKS relative to the DVM and the full UGKS when a parallel calculation is performed. In Table 2, the computa- 
Table 2 Test cases details and computational time

\begin{tabular}{llrrlrrr}
\hline $\begin{array}{c}\text { Test } \\
\text { case }\end{array}$ & M & $\begin{array}{c}\text { Physical } \\
\text { cells }\end{array}$ & $\begin{array}{c}\text { Velocity } \\
\text { cells }\end{array}$ & Solver & Cores & Iterations & $\begin{array}{r}\text { Time, } \\
\text { min }\end{array}$ \\
\hline Shock & 2.8 & 352 & $64 \times 64$ & DVM & 8 & 22480 & 223 \\
Shock & 2.8 & 352 & $64 \times 64$ & GKS & 8 & 14016 & 10 \\
Plate & 4.89 & 24396 & $32 \times 32$ & DVM & 16 & 9178 & 878 \\
Plate & 4.89 & 24396 & $32 \times 32$ & GKS & 16 & 27555 & 363 \\
Wedge & 4.0 & 28016 & $48 \times 32$ & DVM & 64 & 29494 & 1925 \\
Wedge & 4.0 & 28016 & $48 \times 32$ & GKS & 64 & 17623 & 109 \\
\hline
\end{tabular}

tional time for the studied cases is reported and the GKS is found to be from $50 \%$ to $90 \%$ faster than the DVM. This is partially due to the lower number of iterations needed in general by the GKS, but mainly to the time needed per iteration, around 7-10 times smaller than the DVM. Finally, in terms of the memory cost of the GKS, the latter is drastically reduced compared with the DVM and the full UGKS. Indeed, in the DVM and the UGKS, the values of the distribution function need to be stored for each physical cell in the full velocity space while the GKS being employed in the context of a continuum solver requires only the storage of the primitive variables.

Thus, employing the GKS in place of the DVM where the fluid is near thermal equilibrium, the performance of the hybrid solver can be improved in both memory and CPU time requirements, this without compromising the accuracy as shown in subsection 4.1 .

\section{CONCLUDING REMARKS}

In the present work, an analytical GKS has been developed and added to the framework presented in $[23,26,27]$. The prediction of flow fields where rarefied and continuum regions coexist requires the solution of two models; the NS equations and the BTE. Since the methods to solve the BTE are expensive, the reduction of the region where this is strictly required could improve the performance of hybrid simulations. For these reasons, a GKS for near-continuum regime has been proposed.

The scheme has been tested for various cases and Mach numbers proving to produce reliable predictions for near-continuum flows. Moreover, the GKS also proved to be capable of solving more complex three-dimensional flow fields and to couple naturally with a DVM based on the same kinetic model. Regarding the computational time, when compared with a kinetic DVM solver, the nearcontinuum GKS solver was found to be between $50 \%$ and $90 \%$ faster than the 
former. Furthermore, due to the lower number of variables that need to be stored, the GKS is less expensive in terms of memory than the DVM and the full UGKS. This proves that GKS can be a viable way to improve the performance of hybrid simulations maintaining an acceptable level of reliability when used in place of more complex methods for weakly rarefied flows.

\section{ACKNOWLEDGMENTS}

The financial support by the University of Liverpool is gratefully acknowledged. We acknowledge PRACE for awarding us access to SuperMUC at the Leibniz Rechenzentrum (LRZ) in Munich, Germany. The work has also made use of the University of Liverpool cluster "Chadwick" and the N8 HPC facilities provided and funded by the EPSRC and N8 consortium coordinated by the Universities of Leeds and Manchester.

\section{REFERENCES}

1. Vincenti, W., and C. Kruger. 1965. Introduction to physical gas dynamics. New York, NY: John Wiley \& Sons, Inc. 538 p.

2. Bird, G. 2013. The DSMC method. CreateSpace. 300 p.

3. Baranger, C., J. Claudel, N. Hérouard, and L. Mieussens. 2012. Locally refined discrete velocity grids for deterministic rarefied flow simulations. AIP Conference Proceedings. 1501:389-396.

4. Titarev, V., M. Dumbserc, and S. Utyuzhnikov. 2014. Construction and comparison of parallel implicit kinetic solvers in three spatial dimensions. J. Comput. Phys. 256:17-33.

5. Bhatnagar, P., E. Gross, and M. Krook. 1954. A model for collision processes in gases. I. Small amplitude processes in charged and neutral one-component systems. Phys. Rev. 94(3):511-525.

6. Shakhov, E. 1968. Generalization of the Krook kinetic relaxation equation. Fluid Dyn. 3(5):95-96.

7. Rykov, V. 1975. A model kinetic equation for a gas with rotational degrees of freedom. Fluid Dyn. 10(6):959-966.

8. Bourgat, J.-F., P. Le Tallec, and M. Tidriri. 1996. Coupling Boltzmann and NavierStokes equations by friction. J. Comput. Phys. 127:227-245.

9. Le Tallec, P., and F. Mallinger. 1997. Coupling Boltzmann and Navier-Stokes equation by half fluxes. J. Comput. Phys. 136:51-67.

10. Glass, C., and T. Horvarth. 2002. Comparison of a 3-D CFD-DSMC solution methodology with a wind tunnel experiment. NASA/TM-2002-211777.

11. Wang, W.-L., Q. Sun, and I. Boyd. 2002. Towards development of a hybrid DSMCCFD method for simulating hypersonic interacting flows. 8th AIAA/ASME Joint Thermophysics and Heat Transfer Conference. St. Louis, MI. 
12. Schwartzentruber, T. 2007. A modular particle-continuum numerical algorithm for hypersonic non-equilibrium flows. The University of Michigan. PhD Diss.

13. Degond, P., S. Jin, and L. Mieussens. 2005. A smooth transition model between kinetic and hydrodnamic equaions. J. Comput. Phys. 209:665-694.

14. Degond, P., G. Dimarco, and L. Mieussens. 2010. A multiscale kinetic-fluid solver with dynamic localization of kinetic effects. J. Comput. Phys. 229:4907-4933.

15. Deschenes, T., and I. Boyd. 2011. Extension of a modular particle-continuum method to vibrationally exited, hypersonic flows. AIAA J. 49(9):1951-1959.

16. Degond, P., and G. Dimarco. 2012. Fluid simulations with localised Boltzmann upscaling by direct simulation Monte-Carlo. J. Comput. Phys. 231:2414-2437.

17. Xu, K., and J.-C. Huang. 2011. An improved unified gas-kinetic scheme and the study of shock structures. IMA J. Appl. Math. 76:698-711.

18. Liu, S., Y. Pubing, K. Xu, and C. Zhong. 2014. Unified gas-kinetic scheme for diatomic molecular simulations in all flow regimes. J. Comput. Phys. 259:96-113.

19. Xu, K. 2001. A gas-kinetic BGK scheme for the Navier-Stokes equations and its connection with artificial dissipation and Godunov method. J. Comput. Phys. 171:289-335.

20. $\mathrm{Xu}, \mathrm{K}$., and E. Josyula. 2006. Continuum formulation for non-equilibrium shock structure calculation. Commun. Comput. Phys. 1(3):425-450.

21. Xu, K., X. He, and C. Cai. 2008. Multiple temperature kinetic model and gaskinetic method for hypersonic non-equilibrium flow computations. J. Comput. Phys. 227:6779-6794.

22. Xu, K. 2002. Regularisation of the Chapman-Enskog expansion and its description of shock structures. Phys. Fluids 14(4):L17.

23. Steijl, R., and G. Barakos. 2012. Computational fluid dynamics of partially rarefied flows with coupled kinetic Boltzmann/Navier-Stokes methods. ECCOMAS. Vienna, Austria.

24. Colonia, S., R. Steijl, and G. Barakos. 2014. Kinetic models with rotational degrees of freedom for hybrid methods. ECCOMAS: 6th European Conference on Computational Fluid Dynamics. Barcelona, Spain.

25. Andries, P., P. LeTallec, J.-P. Perlat, and B. Perthame. 2000. The Gaussian-BGK Model of Boltzmann equation with small Prandtl number. Eur. J. Mech. B Fluid. 19(6):813-830.

26. Steijl, R., and G. Barakos. 2010. Coupled Navier-Stokes-molecular dynamics simulations using a multi-physics flow simulation framework. Int. J. Numer. Meth. Fl. 62:1081-1106.

27. Steijl, R., and G. Barakos. 2012. Coupled Navier-Stokes/molecular dynamics simulations in nonperiodic domains on particle forcing. Int. J. Numer. Meth. Fl. 69:1326-1349.

28. Colonia, S., R. Steijl, and G. Barakos. 2014. Implicit implementation of the AUSM ${ }^{+}$ and $\mathrm{AUSM}^{+}$up schemes. Int. J. Numer. Meth. Fl. 75(10):687-712.

29. Deschenes, T. 2011. Extension of a modular particle-continuum method for nonequilibrium, hypersonic flows. The Univeristy of Michigan. PhD Diss. 
30. Rykov, V., V. Titarev, and E. Shakhov. 2007. Numerical study of the transverse supersonic flow of a diatomic rarefied gas past a plate. Comp. Math. Math. Phys. 47(1):136-150.

31. Rykov, V., V. Titarev, and E. Shakhov. 2008. Shock wave structure in a diatomic gas based on a kinetic model. Fluid Dyn. 43(2):316-326.

32. Boyd, I. 1990. Rotational-translational energy transfer in rarefied nonequilibrium flows. Phys. Fluids A Fluid 2(3):447-452.

33. Parker, J. 1959. Rotational and vibrational relaxation in diatomic gases. Phys. Fluids 2(4):449-462.

34. Bird, G. 1994. Molecular gas dynamics and the direct simulation of gas flows. 2nd ed. Oxford engineering science ser. Clarendon Press. 484 p.

35. Lordi, J., and R. Mates. 1970. Rotational relaxation in nonpolar diatomic gases. Phys. Fluids 9(4):291-308.

36. Valentini, P., C. Zhang, and T. Schwartzentruber. 2012. Molecular dynamics simulation of rotational relaxation in nitrogen: Implications for rotational collision number models. Phys. Fluids (24):106101:1-23.

37. Jones, F. 1984. NBS TN-1186 - interpolation formulas for viscosity of six gases: Air, nitrogen, carbon dioxide, helium, argon, and oxygen. U.S. Department of Commerce/National Bureau of Standards.

38. Larina, I., and V. Rykov. 2010. Kinetic model of the Boltzmann equation for a diatomic gas with rotational degrees of freedom. Comp. Math. Math. Phys. 50(12):2118-2130.

39. Larina, I., and V. Rykov. 2010. Computation of a rarefied diatomic gas flows through a plane microchannel. Comp. Math. Math. Phys. 52(4):637-648.

40. Colonia, S., R. Steijl, and G. Barakos. 2015. Kinetic models and gas kinetic schemes for hybrid simulation of partially rarefied flows. AIAA Atmospheric Flight Mechanics Conference, AIAA Science and Technology Forum. Kissimmee, FL.

41. Colonia, S., R. Steijl, and G. Barakos. 2016. Kinetic models and gas kinetic schemes for hybrid simulation of partially rarefied flows. AIAA J. 54(4):1264-1276. doi: 10.2514/1.J054137.

42. Deschenes, T., T. Holman, and I. Boyd. 2011. Effects of rotational energy relaxation in a modularparticle-continuum method. J. Thermophys. Heat Tr. 25(2):218-227.

43. Alsmeyer, H. 1976. Density profiles in argon and nitrogen shock waves measured by the absorption of an electron beam. J. Fluid Mech. 74(3):497-513.

44. Candler, G. 1989. On the computation of shock shapes in nonequilibrium hypersonic flows. 27th Aerospace Sciences Meeting. Reno, Nevada.

45. Gnoffo, P., N. Roop, and L. Judy. 1989. Conservation equations and physical models for hypersonic air flows in thermal and chemical nonequilibrium. NASA/TP2867.

46. Tsuboi, N., and Y. Matsumoto. 2005. Experimental and numerical study of hypersonic rarefied gas flow over flat plates. AIAA J. 43(6):1243-1255.

47. Boyd, I., G. Chen, and G. Candler. 1995. Predicting failure of the continuum fluid equations in transitional hypersonic flows. Phys. Fluids 7(1):210-219. 